\title{
Organized Streamwise Vorticity on Convex Surfaces With Particular Reference to Turbine Blades
}

\author{
J. P. Gostelow ${ }^{*}$, A. Mahallati ${ }^{\dagger}$, W. E. Carscallen \\ Institute for Aerospace Research, \\ National Research Council of Canada, Ottawa, K1A OR6, Canada
}

A. Rona ${ }^{\S}$

University of Leicester, Leicester, LE1 7RH, UK

Experiments were conducted on the flow through a transonic turbine cascade and at subsonic speeds past a circular cylinder in cross-flow. These followed extensive work on vortex shedding behind these bodies, displaying the phenomena of energy separation at subsonic speeds; the turbine blades also exhibited exotic vortex-shedding modes in transonic flow. Surface flow visualization was undertaken on the suction surface of the turbine blade and on the circular cylinder. This was effective in providing a time-average mapping of the vortical structures within the blade passage and around the cylinder. The usual phenomena of horseshoe vortices, secondary flows, passage vortices and wall and corner vortices were observed. In addition, and more surprisingly, organized systems of fine-scale streamwise vortices were observed for both cases. Under the influence of the strong favorable pressure gradients on the turbine blade suction surface, the vortices persisted to the trailing edge. For the circular cylinder work, undertaken at an inlet Mach number of 0.5 , the streamwise vortices occupied the forward portion of the cylinder, almost to the 83 degree azimuth, and re-appeared after laminar separation. This streamwise vorticity had been predicted and observed previously for low speed flows, with attendant theories for wavelength. The present results have been compared with the predictions giving reasonable agreement.

\section{Nomenclature}

$\begin{array}{lll}D & = & \text { Diameter of circular cylinder } \\ M_{e} & = & \text { Exit isentropic Mach number } \\ P R & = & \text { Pressure ratio } \\ R e & = & \text { Reynolds number } \\ T u & = & \text { Free-stream turbulence level, \% } \\ \lambda & = & \text { Spanwise wavelength of vortex pairs }\end{array}$

\section{Introduction}

$\mathrm{T}_{\mathrm{s}}^{\mathrm{s}}$ HE authors' previous studies of flows were focused on unsteady surface pressures, wake flows and vortex shedding behind turbine blades and a circular cylinder ${ }^{1,2}$. These displayed the phenomena of energy separation $^{3,4}$ at subsonic speeds and the turbine blades exhibited exotic vortex-shedding modes ${ }^{5-7}$ in transonic flow. In the course of subsequent work, flow visualization was undertaken to investigate the time-averaged flow on the convex surfaces.

Turbine blade designers are quite familiar with the phenomenon of Görtler vorticity, which is thought to occur on the concave pressure surfaces of turbine blades. This organized system of vortices tends to increase heat transfer to the blade surface and also makes the flow and heat transfer very difficult to predict. In this context, the word "organized" is taken to be synonymous with "assembled", "coordinated" or "structured" and carries no imputation of a responsible agent. Organization will be considered to be present if a structure exists on a time-average basis.

\footnotetext{
* Principal Research Officer, GTL, IAR, National Research Council, Ottawa, K1A 0R6, Canada, and Emeritus Professor, Department of Engineering, University of Leicester, Leicester, LE1 7RH, U.K. Member AIAA. Research Officer, GTL, IAR, National Research Council, Ottawa, K1A 0R6, Canada,

\$ Manager of Research and Technology, GTL, IAR, National Research Council, Ottawa, K1A 0R6, Canada. Member AIAA.

$\S$ Lecturer, Department of Engineering, University of Leicester, Leicester, LE1 7RH, U.K. Member AIAA.
} 
It had generally been assumed that streamwise vorticity of this kind was confined to concave pressure surfaces. Examples will be given in this paper which should result in a questioning of the assumption that organized streamwise vorticity is confined to the pressure surface. For a predominantly convex surface, this behavior may be unusual but would be in accordance with the later predictions of Görtler ${ }^{8}$, who postulated instability on a convex surface from the concave streamlines ahead of the leading edge stagnation region, with a resulting upstream zone of flow turbulence and subsequent inflectional instability. A theory giving expected spanwise wavelengths for a circular cylinder was developed by Kestin and Wood in 1970. "Streaky structures" and streamwise vortices have been observed and predicted on flat plates ${ }^{10}$, on compressor blades ${ }^{11,12}$ and on the convex surface of a supercritical airfoil, following an initial concave surface exhibiting Görtler vorticity ${ }^{13}$.

In a study of plane shear layers, Lasheras et al. ${ }^{14}$ have concluded that these are unstable to three-dimensional perturbations in the upstream conditions. The instability results in the formation of organized, three-dimensional vortical structures that propagate and mutually interact under positive and negative strain rates. Although this work was performed in the context of the strong spanwise vortices attending a free shear layer, the conditions are not too dissimilar from those in the leading edge stagnation region of a turbine blade. Diwan and Ramesh ${ }^{15}$ have recently found that the downstream inflectional instabilities observed in separation bubbles have their origins well upstream. They explain streamwise vortices in a separated shear layer by demonstrating that two-dimensional separation is topologically impossible. This seems likely to be a manifestation of the streamwise vorticity discussed in this paper; similar considerations should also apply to two-dimensional attachment at a leading edge stagnation line.

Altogether about ten different vortical mechanisms have been observed in the flow through one turbine nozzle passage of the NRC cascade being studied ${ }^{16}$. This menagerie of vortex encounters involves horseshoe vortices, secondary flows, shock-induced passage vortices and, on the suction surface, streamwise vortices. At the trailing edge, vortex shedding, energy separation, base pressures, and exotic shedding modes are observed. The principal focus of the subject investigations had been on the vortex-shedding behavior. Early measurements on the blading had revealed anomalous distributions of downstream total temperature ${ }^{17}$. These were eventually resolved and shown to result from the vortex shedding through the Eckert-Weise effect ${ }^{4}$. During these early investigations, exotic vortex shedding modes were also observed when running at transonic speeds ${ }^{5-7}$.

This is all in experiments on the flow around one particular turbine nozzle vane in a linear cascade. The diversity of these vortex phenomena is an indication of how little is yet understood about the fundamental internal aerodynamics of turbomachinery blading. If improved accuracy is to be obtained in computational predictions for blade design, greater physical understanding of these phenomena is required. During the investigation attention was also paid to the flow behavior on the blade and end-wall surfaces (Fig. 1) and it is an important aspect of that study, namely streamwise vorticity, that is the focus of the present study. This paper addresses the presence of streamwise vorticity on the suction surface of the turbine blade.

Despite the importance of the problems for turbine blading, these issues are far more general. The cascade had provided an excellent vehicle for study but it was considered important to also reduce the study to the most significant variables and to attempt to address the problems in a relatively generic form. Few published

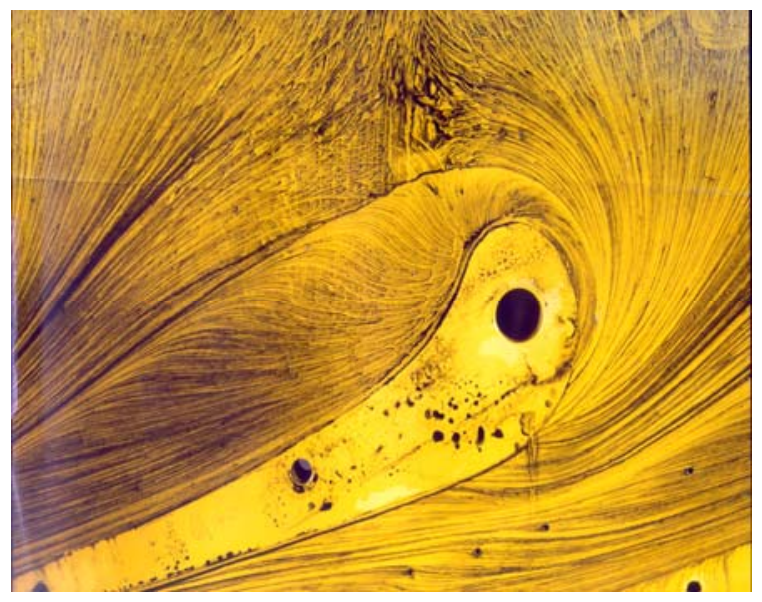

Figure 1. Visualization of the horseshoe vortex.

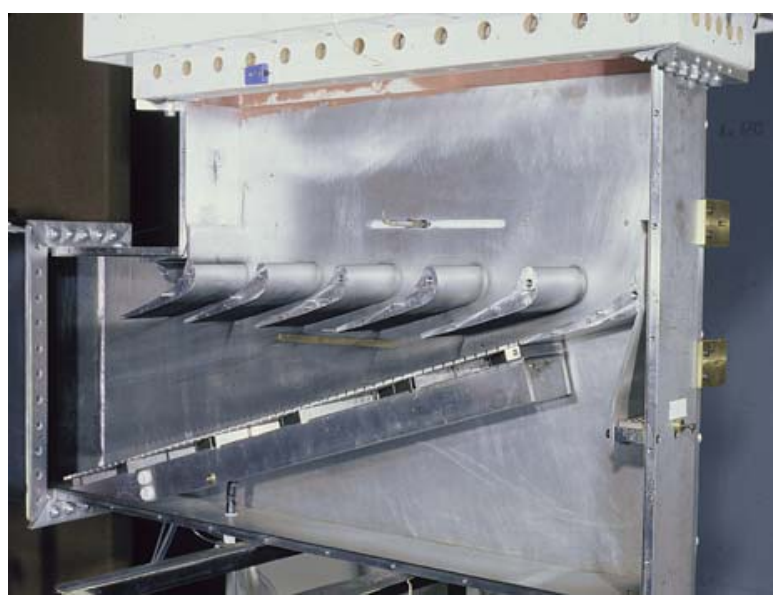

Figure 2. The transonic turbine cascade tunnel.

2

American Institute of Aeronautics and Astronautics 
measurements are available on the circular cylinder at high subsonic speeds, despite the prevalence of such phenomena as severely reduced base pressure and Eckert-Weise energy separation ${ }^{4}$. For this reason it was decided to undertake further tests on an isolated, rigidly mounted circular cylinder in subsonic cross flow. In these investigations, experiments were focused on obtaining reliable measurements at an intermediate subsonic Mach number to obtain the first time-resolved demonstration of energy separation behind a circular cylinder. However additional surface flow visualization was also undertaken on the circular cylinder and was again to provide evidence of organized streamwise vorticity.

\section{Wind Tunnels and Experimental Techniques}

Two wind tunnels at the Institute for Aerospace Research of the National Research Council of Canada (NRC) were used for these experiments. These were the large-scale, continuous flow, transonic linear cascade wind tunnel, shown in Fig. 2 with details in Fig. 3, and the $1.5 \mathrm{~m}$ trisonic blowdown wind tunnel (Fig. 4). Testing in the former tunnel was on a linear cascade of turbine nozzle vanes; in the latter tunnel, it was on the more generic case of a circular cylinder.

\section{A. The Transonic Linear Cascade Tunnel}

In the transonic linear cascade wind tunnel, air was drawn into the test section by a $2 \mathrm{MW}$ compressor having a continuous flow rate of $5 \mathrm{~kg} / \mathrm{s}$. Inlet air to the cascade was drawn through a rectangular bellmouth with a 5:1 elliptical cross section. Since this is an inflow cascade, an increase in the exit Mach number was associated with a corresponding increase in the exit Reynolds number.

Downstream of the cascade, a perforated aluminum tailboard was used to establish the flow periodicity and to eliminate shockwave reflection. This tailboard has proved most effective in reducing undesirable reflections in the downstream flow field. Bypass flaps and tailboards were adjusted to ensure inlet flow uniformity and outlet flow periodicity for each operating condition ${ }^{18}$. The free stream turbulence level, $T u$, of the tunnel at the design condition was $0.2 \%$.

The cascade consisted of a set of 6 airfoils ( 5 passages). The airfoil profile represented the mid-span section of a high-pressure turbine vane originally designed for a highly-loaded, single stage turbine. Details of the stage design and experimental results related to the performance of the vane and the stage have been documented by Williamson and Mustapha ${ }^{17}$. The blade row had a solidity of 1.19 with an aspect ratio of 0.61 . Figure 3 summarizes the geometry of the cascade and shows the inlet and outlet measuring planes. An important feature was that the blades had a circular trailing edge with a diameter of $6.1 \mathrm{~mm}$.

A qualitative measure of flow two-dimensionality in the cascade tunnel was obtained using surface oil-flow visualization. The surface was rendered uniform in color and surface finish by applying white self-adhesive vinyl. A mixture of artist grade linseed oil and powdered lampblack shaded pigment was applied in an extremely thin layer over the suction surface. After letting the tunnel run for about five minutes, resulting in a time-averaged surface visualization, the blade was removed from the cascade and photographed. This technique was used successfully over the whole speed range, including transonic speeds.

Mid-span of a HPT vane, 4.3 times engine size

Correct engine $M_{e}$ of 1.16 at a $P R$ of 2.3

Correct engine $R e$ of $2.13 \times 10^{5}$

Number of Vanes

Axial Chord

6

True Chord

(c) $84.6 \mathrm{~mm}$

Solidity

(c) $186.3 \mathrm{~mm}$

Span

(c/s) $\quad 1.19$

Throat Opening

Trailing Edge Dia.

(h) $112.8 \mathrm{~mm}$

Stagger Angle

Inlet Metal Angle

Incidence Angle

(o) $30.5 \mathrm{~mm}$

(te) $\quad 6.1 \mathrm{~mm}$

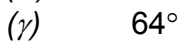

( $\left.\beta_{i}\right) \quad 0^{\circ}$

Outlet Metal Angle

(i) $\quad-10^{\circ}$

( $\left.\beta_{e}\right) \quad 76^{\circ}$

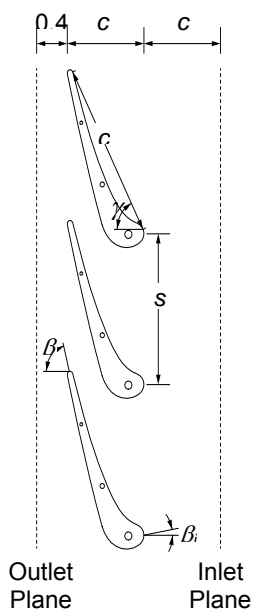

Figure 3. Details and layout of the cascade.

3

American Institute of Aeronautics and Astronautics 


\section{B. The Trisonic Blowdown Tunnel}

For testing on the circular cylinder, the NRC $1.5 \mathrm{~m}$ trisonic blowdown wind tunnel was used. The tunnel draws on extensive high pressure air storage and therefore has a long blowdown time. Blowdown operation resulted in the free stream total temperature dropping slightly over the course of each run as the air in the supply tanks expanded. For this investigation, a two-dimensional flow working section was used, covering the subsonic range. The test section measured $0.381 \mathrm{~m} \times 1.524 \mathrm{~m} \times 3.581$ $\mathrm{m}$. The tunnel was run at a constant Reynolds number of $6.83 \times 10^{5}$.

The model was a circular cylinder with a diameter of $37.26 \mathrm{~mm}$ and a span of $381 \mathrm{~mm}$. The Reynolds number is based on the cylinder diameter and the free stream inflow velocity. The operating Reynolds number range and the run time were dependent on Mach number and were set by adjusting the total and static pressure of the test section. The model was mounted $2.388 \mathrm{~m}$ downstream of the end of the contraction on reinforced mounts in a three-component balance. This was simply used as a mounting rather than a balance and could be rotated through a range of $\pm 55^{\circ}$ at a rate of $15^{\circ}$ per second. Tunnel wall boundary layer control was supplied using ejector-augmented sidewall suction through $0.602 \mathrm{~m} \times 0.452 \mathrm{~m}$ porous plates. The free stream turbulence level, $T u$, at Mach 0.5 was $0.25 \%$.

Following the successful use of the surface flow visualization technique on the transonic cascade tunnel, a similar technique was used on the circular cylinder in the trisonic tunnel at a Mach number of 0.5.

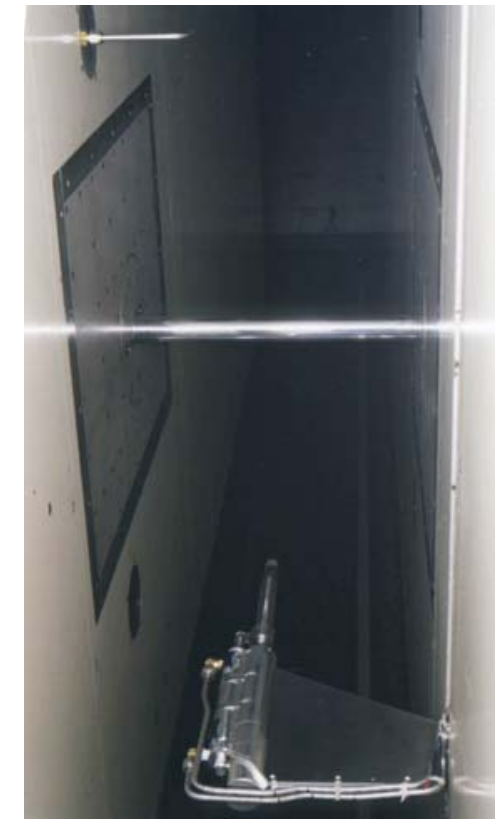

Figure 4. Cylinder and wake probe mounted in trisonic tunnel.

\section{Results From Turbomachinery Blading}

The inlet wall boundary layer occupied $10 \%$ of the span and the leading edge was thick and blunt, giving rise to strong horseshoe vortices and secondary flows. Surface flow visualization was applied, with the intention of establishing the strength of the horseshoe vortex around the leading edge. This resulted in the judgment that on the end walls the influence of the horseshoe vortex was very strong, as shown in Fig. 1. The principal horseshoe vortex around the leading edge had both pressure surface and suction surface components. The pressure surface leg was swept over toward the suction surface, eventually to become the main passage vortex. The static pressure gradient acting on this vortex leg is the same at the end wall as that in the free stream but the velocity is lower in the end wall region. The radius of curvature of the streamlines in the end wall region must also be lower to preserve radial equilibrium. The path the vortex takes in the end wall region therefore has much higher curvature and this accounts for its rapid movement across the passage.

The results of surface flow visualization at three different Mach numbers are presented in Fig. 5. The large numbers on the scale represent percentage axial chord and the small numbers mark static tap locations. In the Mach 0.7 case, a separation region and the secondary flow regions at the two walls are clearly visible. The secondary flow creates a large displacement which affects the streamlines well outside the secondary flow zones. At the mid-span, however, the surface streamlines are all parallel and the flow appears to be two dimensional. In addition to the early separation, the Mach 1.0 case shows a shock impingement and the beginnings of a vortex at each end of this. In the Mach 1.16 case the shock impingement and separation region has moved further downstream with a strong vortex roll-up where the shock impingement meets the side-wall boundary layer.

A close-up view of the vortex formed at the edges of the impinging shock, for the Mach 1.16 case, is given in Fig. 6. The sense of rotation and the back flow into the separated region following the shock are shown clearly. Fine-scale streamwise vortices also show up in the figure. For a predominantly convex surface, this behavior was initially surprising. At the leading edge the suction surface is virtually circular; subsequently much of the suction surface retains a strong convex curvature over the forward portion and is quite flat further downstream.

Surface flow visualization was performed at subsonic and transonic speeds and the surface displayed coherent streamwise vorticity, covering the entire surface and extending to the trailing edge. A close-up view of the visualization from a discharge Mach number of 1.16 is given in Fig. 7. A region between about $80 \%$ and $95 \%$ axial chord is presented. Fine-scale streamwise vortices show up clearly in Fig. 7. For a predominantly convex 


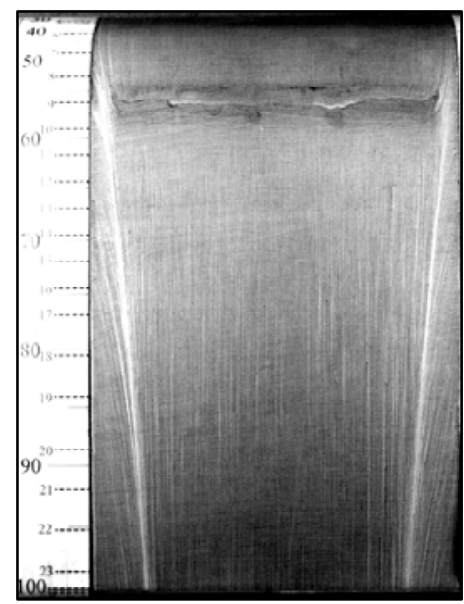

$M_{e}=0.7$

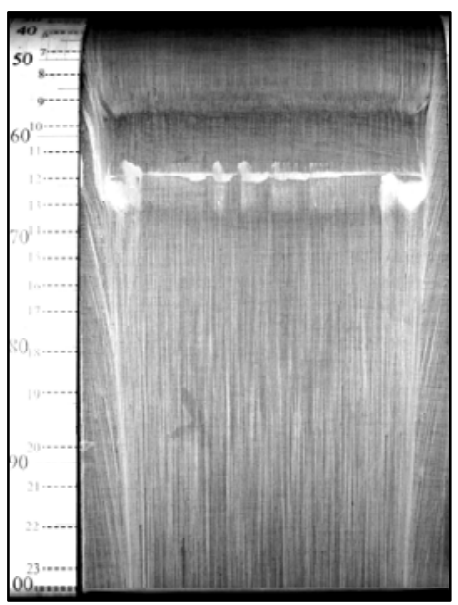

$M_{e}=1.0$

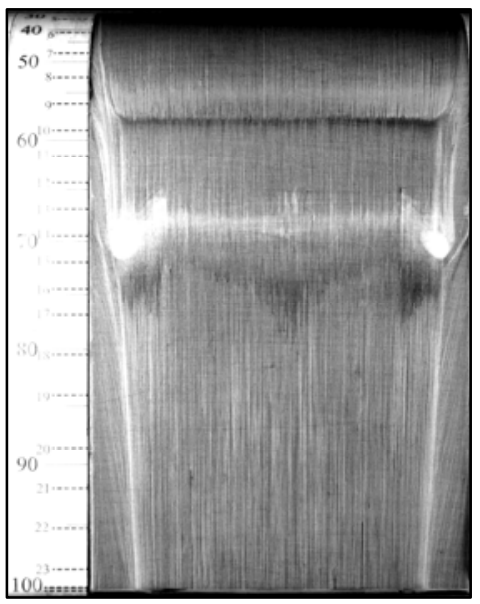

$M_{e}=1.16$

Figure 5. Flow visualization on the suction surface at exit Mach numbers 0.7, 1.0 and 1.16.

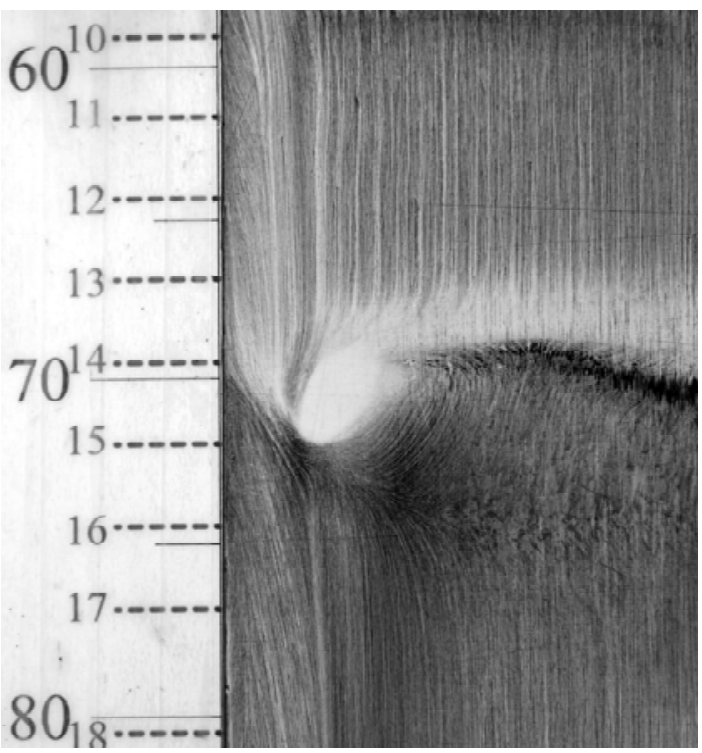

Figure 6. Flow visualization on suction surface at Mach 1.16. Close-up of passage vortex roll-up.

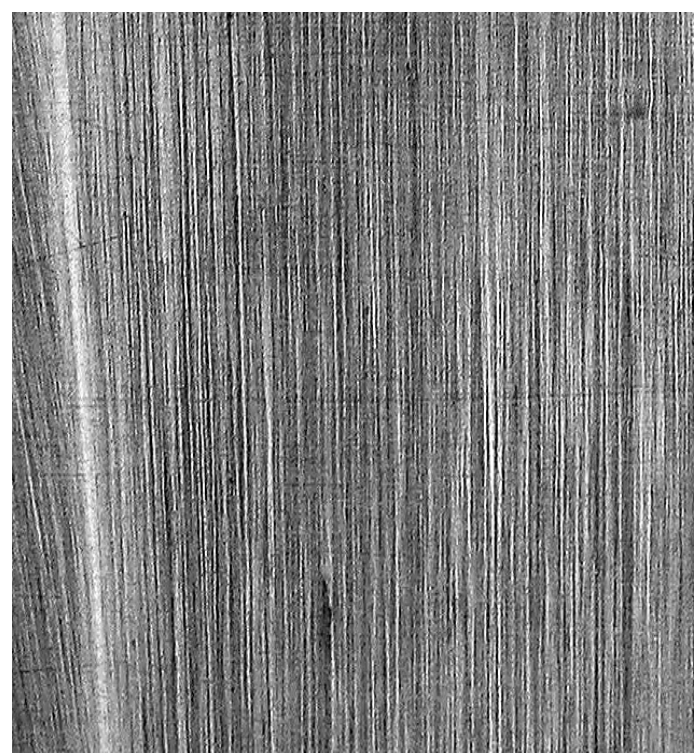

Figure 7. Enlarged visualization of fine-scale streamwise vortices on suction surface.

surface, this behavior is unusual but is in agreement with the later predictions of Görtler ${ }^{8}$, who postulated instability on a convex surface from the concave streamlines ahead of the leading edge stagnation region.

The measurements of the spanwise wavelength of the array of vortices are compared with the predictions of Kestin and Wood ${ }^{9}$ in Fig. 8. Kestin and Wood predicted a theoretical value of pitch wavelength between pairs, $\lambda$, for a cylinder of diameter, $D$, given by:

$$
\lambda=1.79 \pi D \operatorname{Re}^{-0.5}
$$




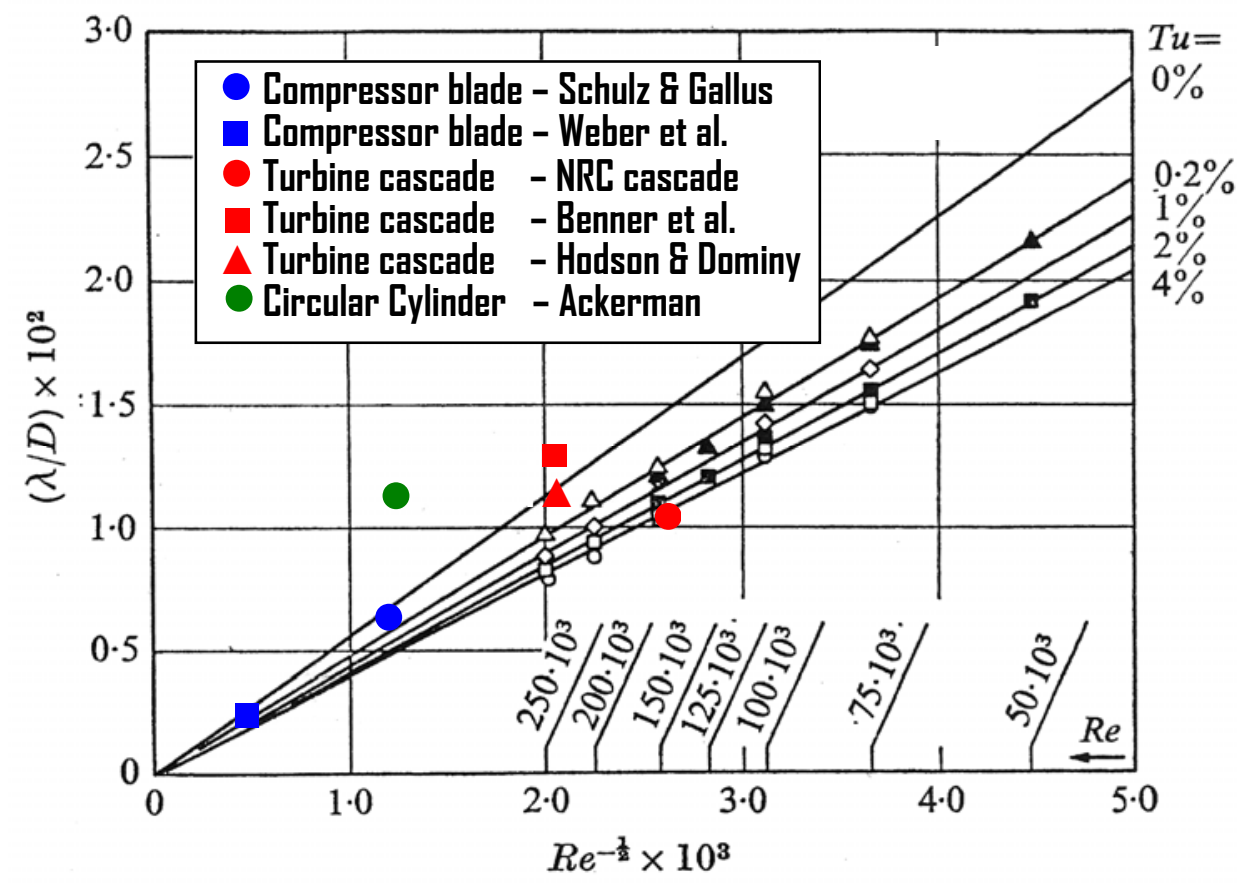

Figure 8. Measurements of wavelength and comparison with Kestin and Wood predictions.

The stability analysis result of Kestin and Wood (Eq. 1) is given by the $T u=0 \%$ line in Fig. 8. They also undertook experimental work on circular cylinders which provided the results for non-zero turbulence levels. It is difficult to locate turbine blade cases on the Kestin and Wood graph as Eq. (1) represents flow past a circular cylinder. The leading edge of the turbine blade is relatively blunt but nevertheless has a high surface curvature which decreases rapidly to near zero further back.

The rapid changes in curvature of the convex surface raise the question of what effective diameter should be applied if comparing with the Kestin and Wood model. Studies of the wall flow visualization and passage geometry resulted in the conclusion that the blade surface curvature at around the $10 \%$ true chord location was quite representative of the curvature of streamlines approaching the suction surface. The diameter of the osculating circle, at the $10 \%$ true chord location on the suction surface, was therefore taken as the value of $D$, when comparing with the Kestin and Wood theory. An average spanwise wavelength, $\lambda$, of $0.55 \mathrm{~mm}$ was measured from the surface visualization photographs for the NRC blade and this is the value used in Fig. 8.

For the NRC blading, the acceleration through the passage is strong. The mass averaged inlet Mach number at the design condition is 0.118 whilst the discharge Mach number is 1.16, giving an order of magnitude difference. Figure 5 shows that the surface flow visualization technique copes very well indeed with both of these very different conditions. It is the flow in the vicinity of the leading edge that determines the susceptibility of the flow to organized streamwise vorticity and, at a local Mach number of 0.118 , the flow dynamics should not be very different from previous observations that were undertaken in low speed flows.

Surface flow visualization photographs have been analyzed from the work of a number of published experiments on blading. When examined in the same way as the surface flow visualization from the NRC cascade, two results from compressor blading (Schulz and Gallus ${ }^{19}$, and Weber at al. ${ }^{11}$ ) and two from turbine blading (Benner et al. ${ }^{20}$, and Hodson and Dominy ${ }^{21}$ ) were accessible; these also gave reasonable agreement with the theory and experiments of Kestin and Wood (Fig. 8). Free-stream turbulence levels for the above cases were all in the range $0.2 \% \leq T u \leq$ $1.2 \%$. In assessing the results, it should be borne in mind that the Mach number regime and model geometry differ from others considered in the published literature. The blades have a very blunt leading edge and are therefore likely candidates to experience flow instability upstream of the stagnation point and the consequent streamwise vorticity. One outcome of these investigations is to establish that organized streamwise vorticity may occur more frequently on convex surfaces, such as turbine blade suction surfaces, than was previously appreciated. Investigations and predictions of flow behavior should be extended to encompass this possibility. If this behavior is at all common, it could have serious implications for turbine aerodynamic and blade cooling design. 


\section{Results from Circular Cylinder}

In the case of the circular cylinder, the geometry is identical with that considered by Kestin and Wood but the Mach number regime is very different. At an inlet Mach number of 0.5 the circular cylinder flow has become critical and is in the intermittent shock regime ${ }^{22}$; this is uncharted territory for considering the streamwise vorticity. The Kestin and Wood analysis was not intended to cope with such conditions and only qualitative agreement should be anticipated in the behavior of the streamwise vorticity.

In the circular cylinder flow, the surface flow visualization work was undertaken at a Mach number of 0.5 . The free-stream turbulence level of the wind tunnel at this Mach number was $0.25 \%$. Although similar techniques were adopted to those used in the cascade tunnel, it had not proved possible to spread the visualization oil as uniformly ${ }^{23}$.

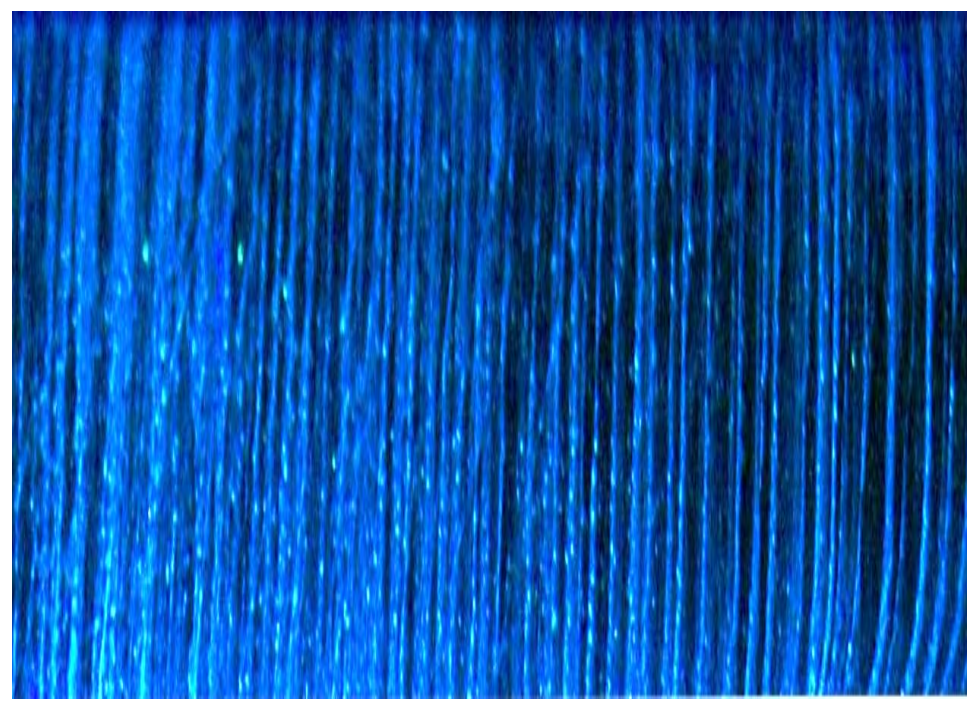

Figure 9. Enlarged visualization of streamwise vortices on circular cylinder.

Similar fine-scale streamwise vortices, observed at Mach 0.5 on the first quadrant of the circular cylinder ${ }^{23,24}$, are presented in Fig. 9. For the circular cylinder the streamwise vortices occupied the forward portion of the cylinder, to around the 83 degree azimuth, and appeared again, at the same spanwise wavelength, after laminar separation. These had a spanwise wavelength, plotted on Fig. 8, somewhat higher than the value predicted by the Kestin and Wood theory.

Although the measured value is almost double that predicted by the theory, this is considered to demonstrate some degree of qualitative compatibility. Possible reasons for the higher measured wavelength are the non-uniform application of the oil, as mentioned previously, and the attainment of locally supersonic flows, alternating from one surface to the other ${ }^{2,22}$.

\section{Conclusions}

Experiments were conducted on the flow through a transonic turbine cascade and at subsonic speeds past a circular cylinder in cross-flow. Surface flow visualization was undertaken on the suction surface of the turbine blade and on the circular cylinder. This was effective in providing a time-average mapping of the vortical structures within the blade passage and around the cylinder. In addition, and more surprisingly, organized systems of fine-scale streamwise vortices were observed for both cases.

In these investigations, on one high-turning turbine nozzle vane cascade, ten distinct modes of vortex formation and shedding were identified. Vortex formations included the expected horseshoe and secondary flow vortices and the surprising organized fine-scale streamwise vorticity on the suction surface. The impact of each of these on turbine performance is potentially harmful. Furthermore, the presence of these disparate vortical structures clearly makes the fluid flow and heat transfer properties of the turbine blading somewhat challenging to interpret and predict.

Under the influence of the strong favorable pressure gradients of the turbine blade suction surface the fine-scale streamwise vortices persisted to the trailing edge. The leading edge geometry is blunt and rather different from the circular cylinder analyzed by Kestin and Wood. In these circumstances, exact predictions by the theory cannot be expected; rather, behavioral similarities are sought. An effective cylinder diameter is applied when applying the Kestin and Wood theory; for turbomachinery blades this was taken to be the diameter of the osculating circle at ten percent true chord. Consistent application of this criterion gave good agreement between flow visualization and prediction for a number of blade geometries. 
Surface flow visualization was performed on the circular cylinder at a Mach number of 0.5 . This is a critical condition with shock waves being formed intermittently on each surface. On the circular cylinder the streamwise vortices occupied the forward portion of the cylinder, to around the 83 degree azimuth and then re-appeared after laminar separation. These streamwise vortices had been predicted and observed previously at low speeds, with attendant theories for spanwise wavelength. The present results, and those of other investigators, have been compared with the predictions giving a reasonable measure of qualitative compatibility. Stability analysis originally foreshadowed the experimental observations and could usefully be extended to account for representative blading geometries and flow conditions.

The outcome of these investigations is to establish that organized fine-scale streamwise vorticity may occur more frequently on convex surfaces, such as turbine blade suction surfaces, than hitherto appreciated. Experimental investigations, and computational predictions of flow behavior, should be extended to encompass that possibility. If the flow behavior described in this paper is at all common it could have implications for turbine aerodynamic and blade cooling design.

\section{Acknowledgments}

The authors are indebted to the National Research Council of Canada (NRC) for the use of the transonic cascade tunnel and the U-66 $1.5 \mathrm{~m}$ trisonic wind tunnel. Appreciation is expressed to Paul Hunt, Jon Ackerman and Stephen Andrews for their excellent flow visualization work.

\section{References} 1996 ,

${ }^{1}$ Carscallen, W. E., Fleige, H. U., and Gostelow, J. P., "Transonic Turbine Vane Wake Flows," ASME Paper 96-GT-419,

${ }^{2}$ Ackerman, J. R., Gostelow, J. P., Rona, A., and Carscallen, W. E., "Measurements of Fluctuating Pressures on a Circular Cylinder in Subsonic Cross Flow," AIAA Journal, Vol. 47, No. 9, 2009, pp. 2121-2131.

${ }^{3}$ Eckert, E. R. T., and Weise, W., "Messungen der Temperatureerteilung auf der ober Fläche Schnell Angeströmter Unbeheizter Körper," Forshg. Ing. Wesen. Vol. 13, 1943, pp. 246-254.

${ }^{4}$ Carscallen, W. E., Currie, T. C., Hogg, S. I., and Gostelow, J. P., "Measurement and Computation of Energy Separation in the Vortical Wake Flow of a Turbine Nozzle Cascade," Journal of Turbomachinery, Vol. 121, No.4, 1999, pp. 703-708.

${ }^{5}$ Carscallen, W. E., and Gostelow, J. P., "Observations of Vortex Shedding in the Wake from Transonic Turbine Nozzle Vanes," Proc. ISROMAC-5, Hawaii, 1994, pp 153-169.

${ }^{6}$ Williamson, C. H. K. and Roshko, A., "Vortex Formation in the Wake of an Oscillating Cylinder," Journal of Fluids and Structures, Vol. 2, 1988, pp. 355-381.

${ }^{7}$ Ponta, F., and Aref, H., "Numerical Experiments on Vortex Shedding from an Oscillating Cylinder," Journal of Fluids and Structures, Vol. 22, 2006, pp. 327-344.

${ }^{8}$ Görtler, J., "Three-Dimensional Instability of the Stagnation Point Flow with Respect to Vertical Disturbances," (in German). In 50 Years of Boundary Layer Research, ed. Görtler and Tollmien, Vieweg, Braunschweig, 14, 17, $304-14.1955$.

${ }^{9}$ Kestin, J., and Wood, R. T., "On the Stability of Two-Dimensional Stagnation Flow," Journal of Fluid Mechanics, Vol. 44, 1970, pp. 461-479.

${ }^{10}$ Alfredsson, P. H., and Matsubara, M., "Streaky Structures in Transition," Transitional Boundary Layers in Aeronautics, R.A.W.M. Henkes and J.L. van Ingen, eds. North-Holland, Amsterdam, 1996, pp. 373-386.

${ }^{11}$ Weber, A., Schreiber, H-A., Fuchs, R., and Steinert, W., "3D Transonic Flow in a Compressor Cascade with Shock-Induced Corner Stall," Journal of Turbomachinery, Vol. 124, No. 3, 2002, pg. 358.

${ }^{12}$ McMullan, W. A., and Page, G. J., "Large Eddy Simulation of a Controlled Diffusion Cascade," Private communication, under review, 2009.

${ }^{13}$ Mangalam, S. M., Dagenhart, J. R., Hepner, T. E. and Meyers, J. F., "The Görtler Instability on an Airfoil," Proc. AIAA 23rd Aerospace Sciences Meeting, Reno, AIAA-85-0491, Jan. 1985.

${ }^{14}$ Lasheras, J. C., Cho, J. S., and Maxworthy, T., "On the Origin and Evolution of Streamwise Vortical Structures in a Plane, Free Shear Layer," Journal of Fluid Mechanics, Vol. 172, 1986, pp. 231-258.

${ }^{15}$ Diwan, S. S., and Ramesh, O. N., "On the Origin of the Inflectional Instability of a Laminar Separation Bubble," Journal of Fluid Mechanics, Vol. 629, 2009, pp. 263-298.

${ }^{16}$ Carscallen W. E., Gostelow J. P., and Mahallati, A., "Some Vortical Phenomena in Flows over Transonic Turbine Nozzle Vanes Having Blunt Trailing Edges," Proc. ISABE 2009-1216, Montreal.

${ }^{17}$ Williamson, R. G., and Moustapha, S. H., "Annular Cascade Testing of Turbine Nozzles at High Exit Mach Numbers," Journal of Fluids Engineering, Vol. 108, No. 198, pp. 313-320.

${ }^{18}$ Gostelow, J. P., Mahallati, A., Andrews, S. A., and Carscallen, W. E., "Measurement and Computation of Flowfield in Transonic Turbine Nozzle Blading with Blunt Trailing Edges,” ASME Paper GT2009-59686, IGTI, Orlando, June 2009. 
${ }^{19}$ Schulz, H. D. and Gallus, H. D., (1988), "Experimental Investigation of the Three-Dimensional Flow in an Annular Compressor Cascade," Journal of Turbomachinery, 110, 467-478.

${ }^{20}$ Benner, M. W., Sjolander, S. A., Moustapha, S. H., "Measurements of Secondary Flows in a Turbine Cascade at OffDesign Incidence," ASME Paper 97-GT-382. 1997.

${ }^{21}$ Hodson, H. P. And Dominy, R. G.,"The Off-Design Performance Of A Low-Pressure Turbine Cascade," Journal of Turbomachinery, Vol. 109, 1987, pp. 201-209.

${ }^{22}$ Zdravkovich, M. M., "Flow Around Circular Cylinders, ” Oxford University Press, 1997.

${ }^{23}$ Ackerman, J. R., "Unsteady Energy Separation and Base Pressure Distributions in Subsonic Crossflow around a Circular Cylinder," PhD thesis, University of Leicester, 2004.

${ }^{24}$ Gostelow, J. P., Carscallen, W. E., Ackerman, J. R., and Rona, A., "Measurements of Base Pressure and Energy Separation on a Circular Cylinder in Subsonic Cross Flow," Proc. AIAA Aeroacoustics Conference, Miami, AIAA-2009-3259, May 2009. 